'Miembro Honorario, Academia Chilena de Medicina.

${ }^{2}$ Miembro Correspondiente, Academia Chilena de Ciencias, Instituto de Chile. Santiago, Chile.

Trabajo no recibió financiamiento.

El autor declara no tener conflictos de interés.

Recibido el 10 de junio de 2019, aceptado el 4 de septiembre de 2019.

Correspondencia a: Felipe Cabello, MD cabello@nymc.edu

\section{Julio Escámez Contreras y su original mural Historia de la Medicina y de la Farmacia en Chile}

\author{
FELIPE CABELLO C. ${ }^{1,2}$
}

\section{Julio Escámez Contreras and his masterful mural History of the Medicine and Pharmacy in Chile}

Julio Escámez Contreras was a highly creative, skilled and versatile Chilean painter who painted in Chile from 1940 to 1974 when he went into exile to Costa Rica and died there in 2015. In 1953-54, Escámez painted a large mural in a private pharmacy in the city of Concepcion, Chile, The History of Medicine and Pharmacy in Chile. This mural describes the origins and development of medicine and pharmacy in Chile, placing that origin in the medicinal activities of the mapuche indigenous population. The mural consists of three sections, each painted on the upper segment of three adjacent walls in the pharmacy, with each section focused on one period in the evolution of medicine and pharmacy in Chile. The first section is devoted to indigenous medical practices including its pharmacopeia and religious practices, the second describes medical approaches during colonial times, still with strong indigenous components but also with indigenous and Catholic hybrid religious elements, while the third depicts modern medicine, including chemistry, anatomical, physical and pharmacological activities complemented with public health components such as nursing, vaccination and health education. Read from left to right, the mural provides a highly lively, accurate and valid depiction of the evolution of medicine and pharmacy in Chile. Escámez' artistry and skill in the use of perspective, color, landscape, architecture and Chilean subjects, including real life individuals, produces a typical Chilean mural. However, his originality and consummate use of a non-verbal visual language delivers a more universal message, one that helps to explain the repeated efforts, of the government responsible for his exile, to destroy some of the works produced by him.

(Rev Med Chile 2019; 147: 1190-1198)

Key words: Chile; History; Medicine; Pharmacy; Population Groups.
"La originalidad es la profundidad de su expresión, ser original es ser auténtico y no cambiar de estilos constantemente de acuerdo al mercado".

Julio Escámez C.

ecientemente se ha despertado un gran interés en Europa y Norte América por lo que fuera llamado "arte degenerado" (entartete künst en alemán), que fue el arte, prohibido a menudo confiscado y destruido por el gobierno de Hitler en Alemania ${ }^{1-3}$. En este país antes de la Gran Guerra y durante la república de Weimar, el arte pictórico estaba en la vanguardia del modernismo mundial con movimientos que incluían el Puente, el Expresionismo, el Jinete Azul, y a la Nueva Objetividad ${ }^{1-7}$, y pintores como Ernst L. Kirchner, Franz Marc, Max Ernst, Paul 
Klee, Elfriede Lohse-Wächtler, Emil Nolde, Paula Modersohn-Becker y Maria Caspar-Filser ${ }^{1-7}$. Posteriormente de la ascensión de Hitler al poder, los teóricos de su gobierno, Joseph Goebbels y Alfred Rosenberg, establecieron las bases para clasificar a diferentes expresiones artísticas como degeneradas y carentes de un legítimo espíritu germano y planear su destrucción ${ }^{1-7}$.

La prohibición y la destrucción de piezas de arte, sin embargo, no es monopolio del nazismo, en 1907 en la Universidad de Viena los murales representando a la Jurisprudencia, a la Medicina y a la Filosofía, comisionados por la misma universidad al pintor Gustav Klimt, fueron tildados de pornográficos y nunca fueron instalados, desapareciendo finalmente durante la Segunda Guerra Mundial $^{8,9}$. El mural de Diego Rivera pintado en el Rockefeller Center, Nueva York, titulado "El Hombre en la Encrucijada", fue destruido por orden de la familia Rockefeller en 1933, pero reproducido fielmente por el pintor en el Palacio de Bellas Artes de México en $1934^{10,11}$. Chile tampoco ha estado inmune a destruir obras de arte consideradas ofensivas para el orden establecido y juzgadas carentes de suficiente devoción patria, como lo demuestra la obra del pintor nacional Julio Escamez ${ }^{12-19}$.

Dos murales de este eximio pintor (Figura 1), representante de una época saliente de la cultura chilena, "El principio y el fin", (1973) en la Municipalidad de Chillán y "El hombre ante el micro y el macrocosmo", (1960?) en el Liceo de Hombres Enrique Molina, Concepción, fueron destruidas después de 1973 por orden de las autoridades militares de la época ${ }^{12-19}$. La original obra del pintor de la cual Pablo Neruda dijera, que era "de una luminosa honestidad" y en la cual "vemos como que se han reconocido las esencias más extraordinarias de la creación, que tienen la quietud de la profundidad, de la verdad, de la poesía. Admiro en él tantos aspectos de una presencia creadora que es como una flor en su unidad maravillosa.", llego a ser prohibida y el pintor hubo de exiliarse en Costa Rica donde falleciera el año 2015.

\section{Homenaje (Historia) de la Medicina y de la Farmacia en Chile}

Para el autor de este artículo, interesado en las relaciones entre medicina y arte, fue una sor- presa saber que además del mural de Mario Toral "Alegoría de la Medicina" (2005), en la Facultad de Medicina de la Universidad de Chile, existía en Chile otro mural que tenía como tema la historia de la medicina y de la farmacia en el país; mural pintado entre 1957-58 y declarado Monumento Nacional en $2015^{12,16,18}$. El mural está ubicado en la antigua Farmacia Maluje, calle Tucapel 676, de Concepción (ahora Droguería Alemana). Este fresco tiene una superficie de aproximadamente $200 \mathrm{~m}^{212,16,18}$, está dividido en tres secciones y está pintado en la parte superior de las murallas de la farmacia que tienen doble altura; cada sección representaría a una diferente etapa histórica de la medicina y de la farmacia en Chile $e^{12,16,18}$.

\section{Primera sección}

La primera sección izquierda (Figura 2) está dividida en dos áreas que representan, a la medicina y a la farmacia mapuche, la que siguieran practicándose durante la Colonia y hasta hoy en día ${ }^{12,16,18}$. En ella, una mujer mapuche, ataviada como machi, colecciona hojas de plantas medicinales a la izquierda de un boldo florido, árbol cuyos productos aún son usados en la farmacopea mapuche y en la medicina chilena alternativa ( $\mathrm{Fi}-$ gura 2a) ${ }^{18,19}$. Al lado derecho del boldo camina un niño descalzo con un poncho corto y un cayado de madera como el cual se juega a la chueca ${ }^{12,18}$.

La parte media de esta sección está ocupada por varios jinetes procediendo de varias direcciones, que se dirigen a un nguillatún, representado en el ángulo superior derecho, donde pueden verse el sacrificio animal, la colección ritual de su sangre y la reunión de mapuches alrededor del rewe sagrado tallado como escalera ${ }^{12,18,19}$. Los jinetes, las mujeres

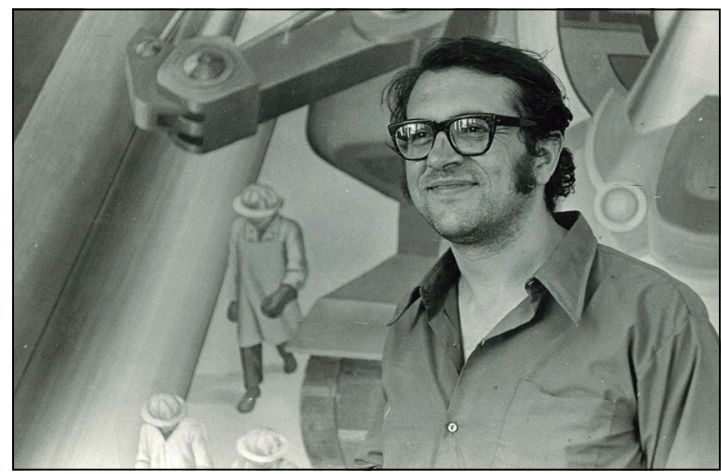

Figura 1. Julio Escámez C. 1973 


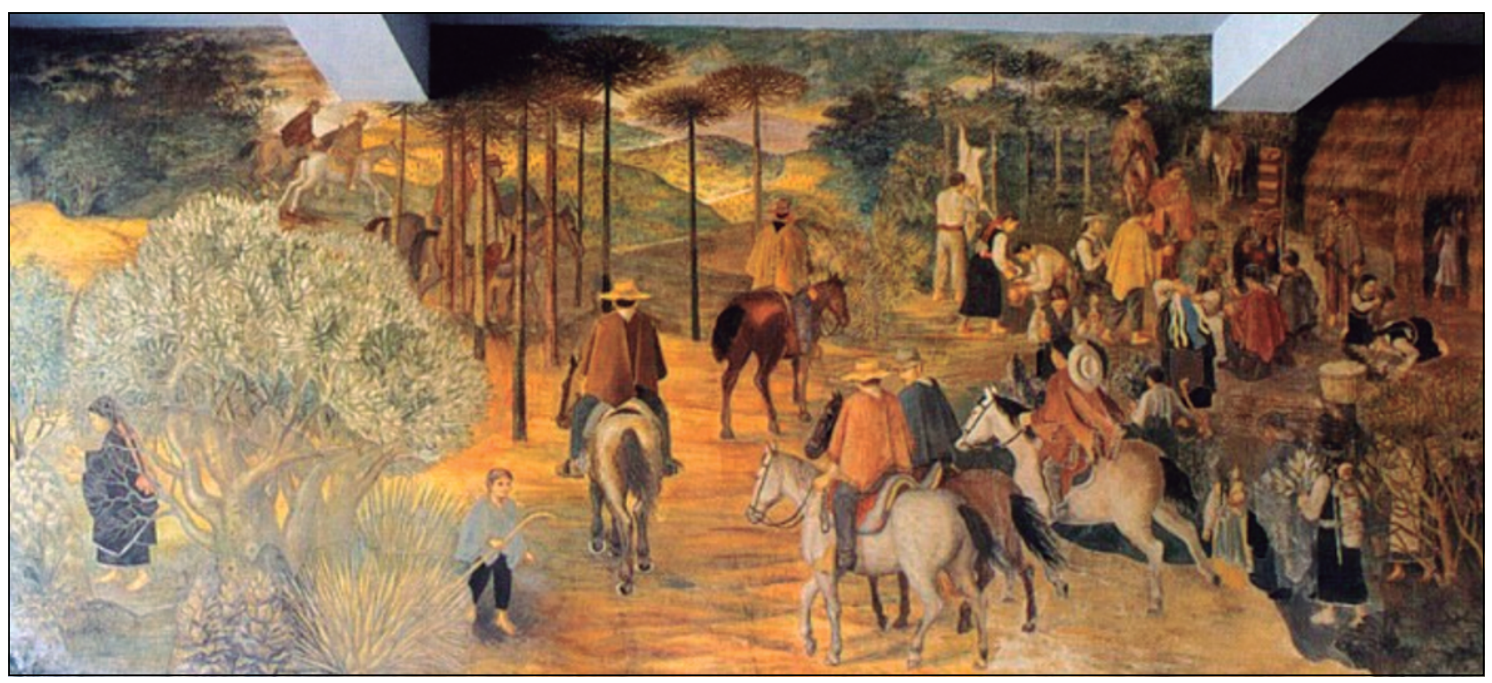

Figura 2. Medicina mapuche.

mapuches con atuendos típicos caminando desde el ángulo inferior derecho hacia el guillatún, los niños jugando en frente de una ruca y una figura femenina ágil en el dintel de esta, le imprimen al fresco importante dinamismo y vigor ${ }^{12,18,19}$.

Realismo es manifestado por el uso de la perspectiva en la representación de las araucarias y de las colinas del fondo de la pintura y la paleta de colores verdes y ocres, que dan una sensación de espacio tridimensional, fundiéndose naturalmente la pintura con el cielo raso y las vigas de la construcción $1^{12,18,19}$. A pesar de la presencia de los caballos, esta primera sección representa a la medicina mapuche precolonial autóctona y en ella el pintor concilia adecuadamente el uso de fuentes farmacológicas naturales y de la religión, en su aplicación al proceso curativo ${ }^{12,18,19}$.

\section{Segunda sección}

Esta sección (Figura 3) está también dividida en dos áreas con diferentes actividades llevadas a cabo en ellas en edificios, siendo el edificio de la izquierda un convento u hospital y el de la derecha la casa de una hacienda o fundo ${ }^{12,18,19}$. Ambas construcciones tienen aspectos mediterráneos europeos dado la composición de sus paredes, techos y arcos y también por el uso de colores ocres, verdes y naranjas, demostrando la influencia hispánica en Chile ${ }^{12,18,19}$. En el convento/hospital se desarrolla una intensa actividad de contenido terapéutico que incluye la presencia de un herba-

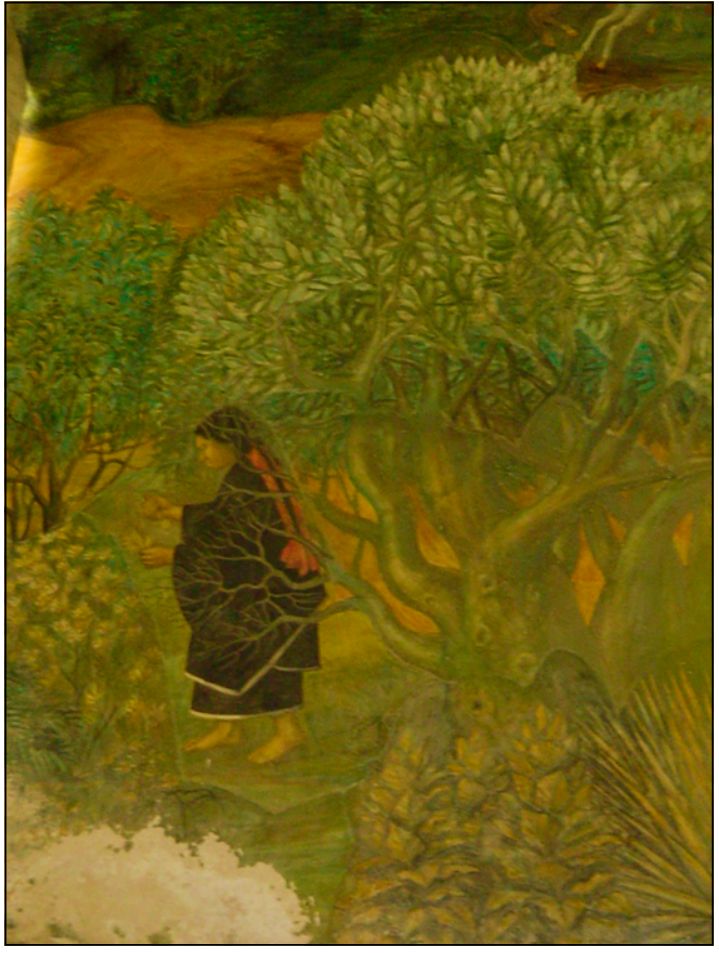

Figura 2a. Medicina mapuche, detalle.

rio y la colección de hierbas por una religiosa, la preparación de extractos botánicos en una mesa con un mortero y el subsecuente almacenamiento de estos extractos naturales y otros fármacos, en 


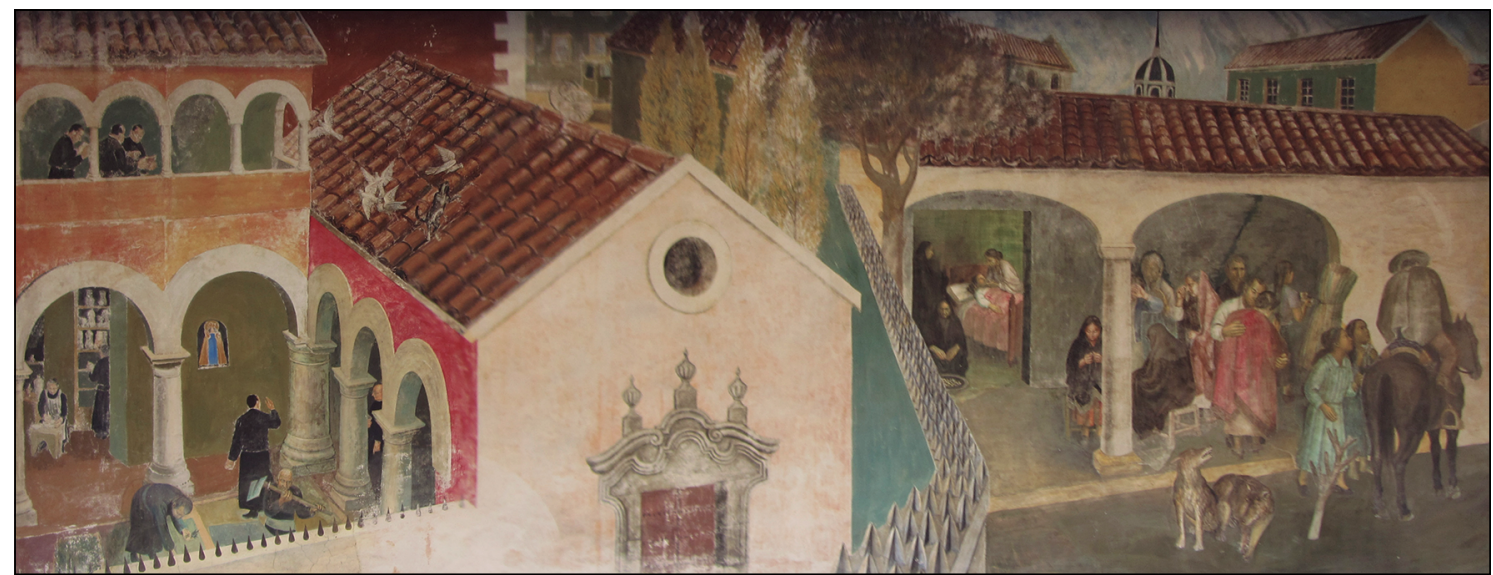

Figura 3. Medicina colonial.

frascos de porcelana ordenados en alacenas y con etiquetas que los identifican (Figura 3a). El uso de la oración y de la música en el proceso curativo es sugerido por la presencia de una imagen religiosa de la Virgen en una muralla y la presencia de un religioso usando un instrumento similar a un oboe o fagot ${ }^{12,18,19}$. En un segundo piso, clérigos leyendo y conversando con documentos en la mano, sugieren a estudiosos de la medicina y de la farmacopea y las palomas podrían ser signos de la paz necesaria para el proceso curativo y su vuelo, complementa la afanosa actividad realizada en el patio del convento/hospital ${ }^{12,18,19}$.

La sección de la derecha contiene tres planos, siendo el plano del fondo un dormitorio donde a un enfermo/a una asistente le levanta la cabeza para ayudarle a la ingestión de una poción, al mismo tiempo que sobre un brasero una curandera quema hierbas con actividades curativas y con un probable simbolismo religioso (Figura $3 \mathrm{~b})^{12,17}$. En el medio, en una galería abierta de la casa, hay un grupo doliente donde una mujer con un rosario o un collar de cuentas está orando mientras otras personas afligidas lloran, y se consuelan mutuamente, dándole al grupo un gran sentido de congoja $^{12,18,19}$. Fuera de la casa, dos mujeres hablan con un jinete pidiéndole tal vez traer a un médico, medicinas o un sacerdote para que administre los últimos ritos a la persona enferma. Los dos perros retozando completan la ruralidad de las escenas de esta sección que, como la primera sección, pareciera mostrarnos aun la dualidad indígena natural y religiosa del proceso curativo.

\section{Tercera sección}

En esta sección la medicina moderna irrumpe en plenitud con actividades propias de laboratorios de farmacología y fisiología y procedimiento preventivos como son la vacunación y la

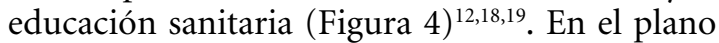
inferior izquierdo de ella laboratoristas realizan procedimientos químicos con matraces y buretas y otro personaje clasifica y pesa sustancias químicas contenidas en papeles sobre una mesa ${ }^{12,18,19}$. La figura detrás de ellos con barba, es la del profesor Alejandro Lipschüz, profesor de la Universidad de Concepción y Premio Nacional de Ciencias (1969), también detrás se ve un modelo del cuerpo humano y un diagrama que correspondería al átomo ${ }^{12,18,19}$. En el plano superior, alrededor de una mesa. mujeres llevan a cabo tareas de laboratorio y detrás de ellas un hombre vigila un complejo equipo de destilación, siendo todas estas actividades supervisadas por la figura del químico farmacéutico Daniel Belmar, profesor de la U. de Concepción y además novelista de renombre (Roble Huacho, 1947; Coiron, 1950) ${ }^{12,18,19}$.

Los procesos de vacunación y de educación sanitaria están representados a la derecha de esta sección de una manera muy gráfica (Figura 4a), en ella enfermeras y auxiliares llevan a cabo estas acciones preventivas en el público, en el cual se mezclan expectantes, las diferentes edades, los sexos y las clases sociales y estarían representadas Violeta Parra, Orietta Escámez hermana del pintor y Mariam Contreras Maluje, hija de los propietarios de la farmacia ${ }^{12,18,19}$. Este plano hace 


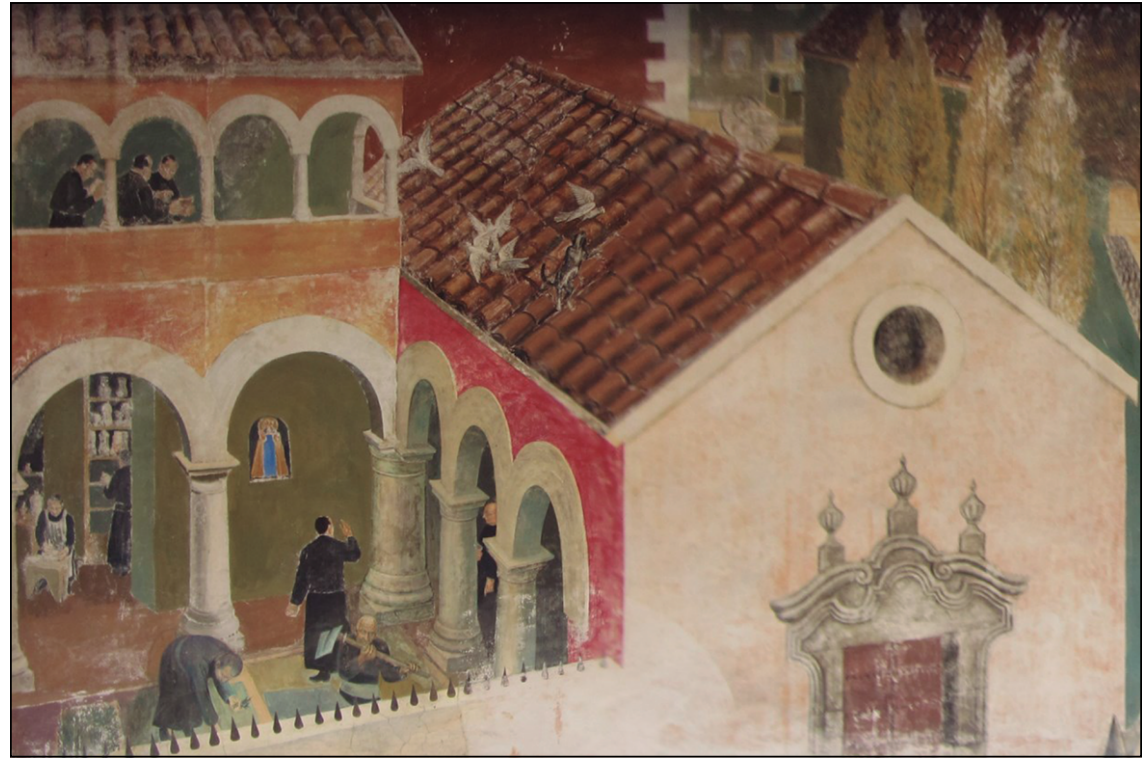

Figura 3a. Medicina colonial, detalles.

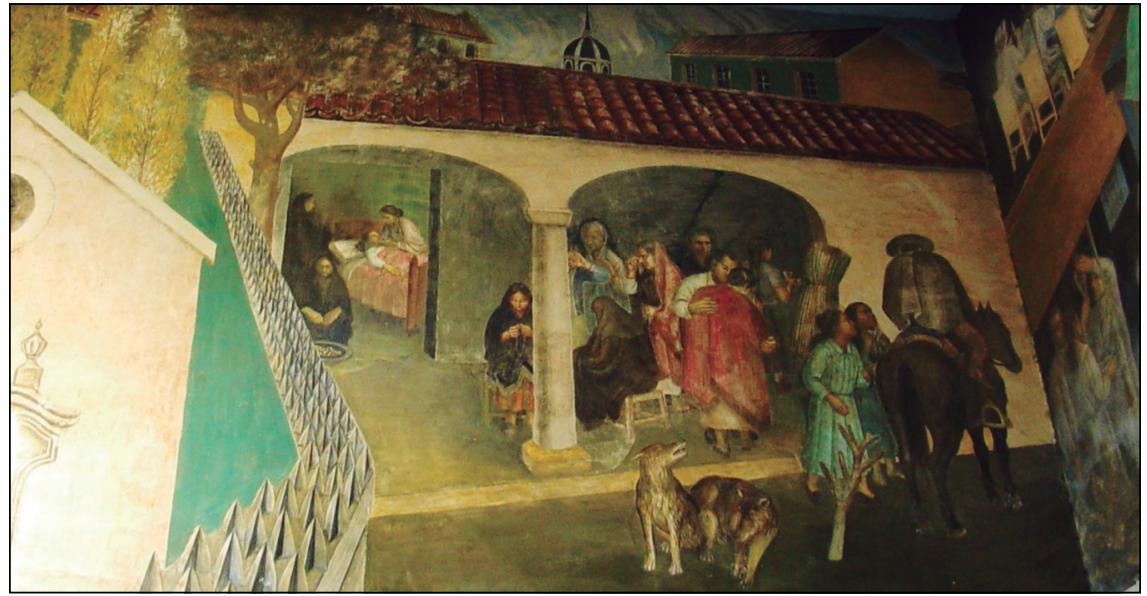

Figura 3b. Medicina colonial, detalles. recordar las campañas masivas de vacunación contra la viruela y la tuberculosis llevadas a cabo por el personal del servicio Nacional de Salud en la época en que se pintó el cuadro ${ }^{12,18,19}$. Las dos enfermeras caminando ágilmente hacia la derecha parecen actuar como puente entre el mundo de la investigación representado a la izquierda y el mundo de la aplicación práctica de ella en la salud pública, a la derecha; ilustrado esto además porque la enfermera de la derecha pareciera llevar en sus manos un producto de esta investigación (Figura 4b). Debajo de las enfermeras hay un grupo de cuatro personas conversando, una leyendo un diario con una representación del hongo de una explosión atómica y la otra sosteniendo una paloma entre sus manos como símbolos de los usos bélicos y pacíficos de la energía atómica ${ }^{12,18,19}$. El personaje solitario que fuma y usa un sombrero de vaquero (Figura 4b), representa al físico Robert J. Oppenheimer, uno de los inventores de la bomba atómica, que fuera perseguido políticamente en la década de los 50 en EE.UU. por sus supuestas simpatías comunistas y su oposición a la creación de la bomba de hidrogeno, quien fuera fumador empedernido, usara sombreros de ala y falleciera víctima de un cáncer faríngeo en 1962 12,18,19,20. 


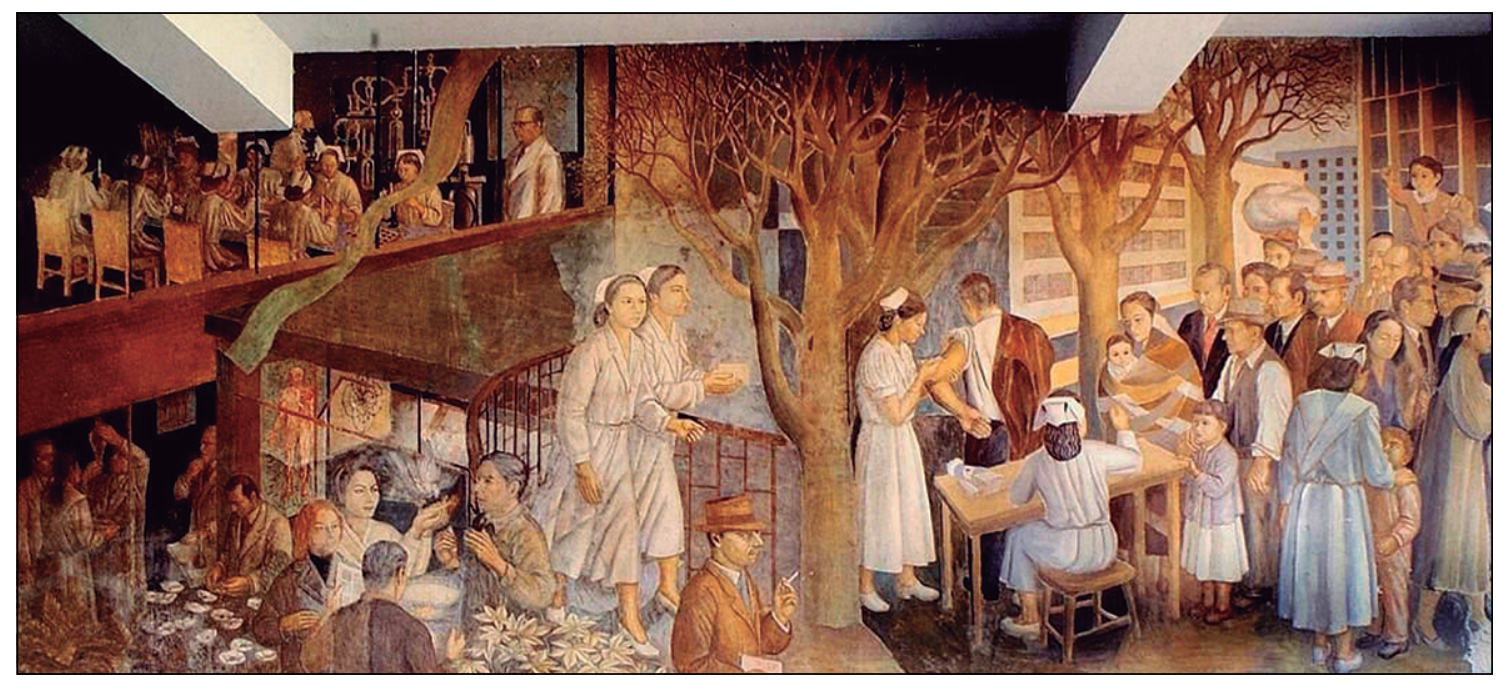

Figura 4. Medicina moderna.

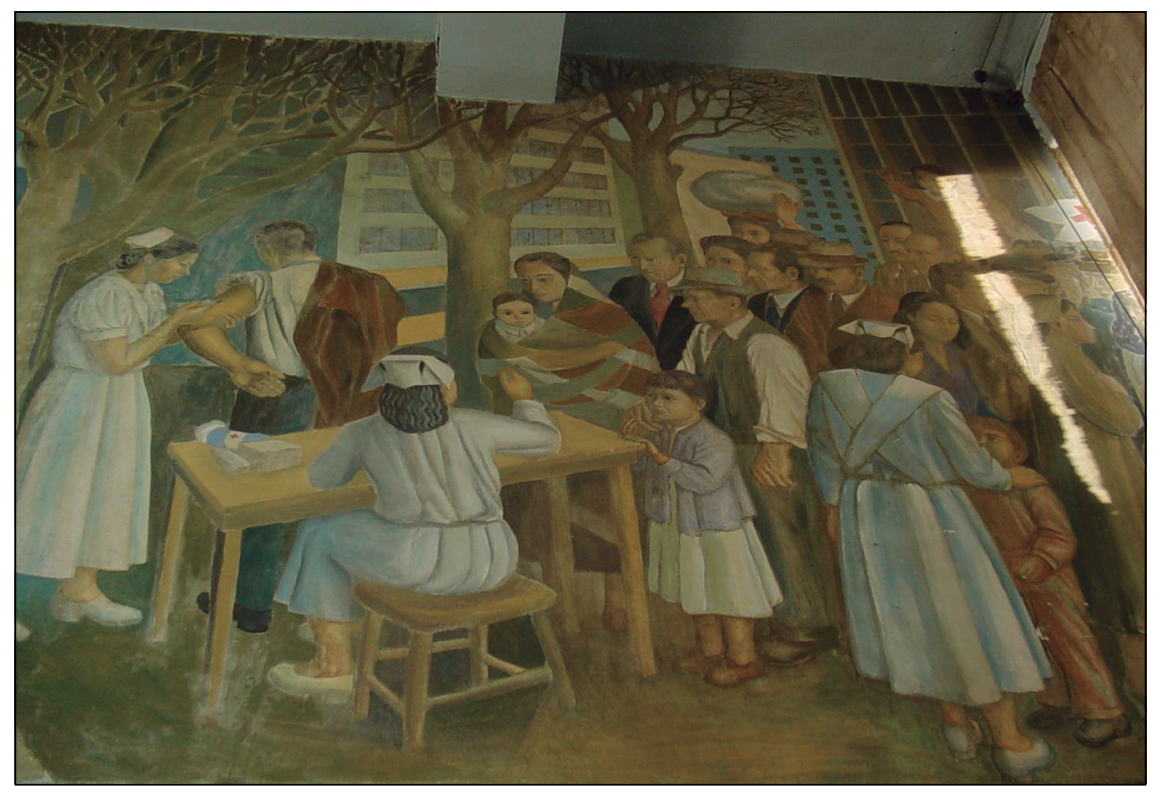

Figura 4a. Medicina moderna detalles.

\section{El pintor Julio Escámez C.}

La lectura de la obra "Los sueños del pintor" (2012) del Premio Nacional de Literatura (2007) Jose Miguel Varas basadas en entrevistas a su amigo Julio Escámez nos ayuda a entender el arte del pintor de una manera más prolija y también apreciar su tremenda originalidad y su gran versatilidad, adquirida a través de años de aprendizaje y de intensa practica ${ }^{21}$. Escámez fue un viajero frugal y sempiterno que recorrió extensivamente Chile,
Latino América, Europa y Asia, incluyendo a Sri Lanka, la India, Japón, la China y Viet Nam, en las condiciones más difíciles, debido a una falta crónica de dinero que no menguaba su curiosidad y su optimismo $^{19}$. Escámez desde su infancia rural en las provincias de Arauco (Antihuala donde naciera en 1925 y Cañete) y en la del Bío Bío, demostró una gran habilidad para el dibujo y las actividades plásticas, además de una gran curiosidad por su entorno geográfico y por la riqueza de los personajes singulares de su niñez provinciana ${ }^{21}$. 


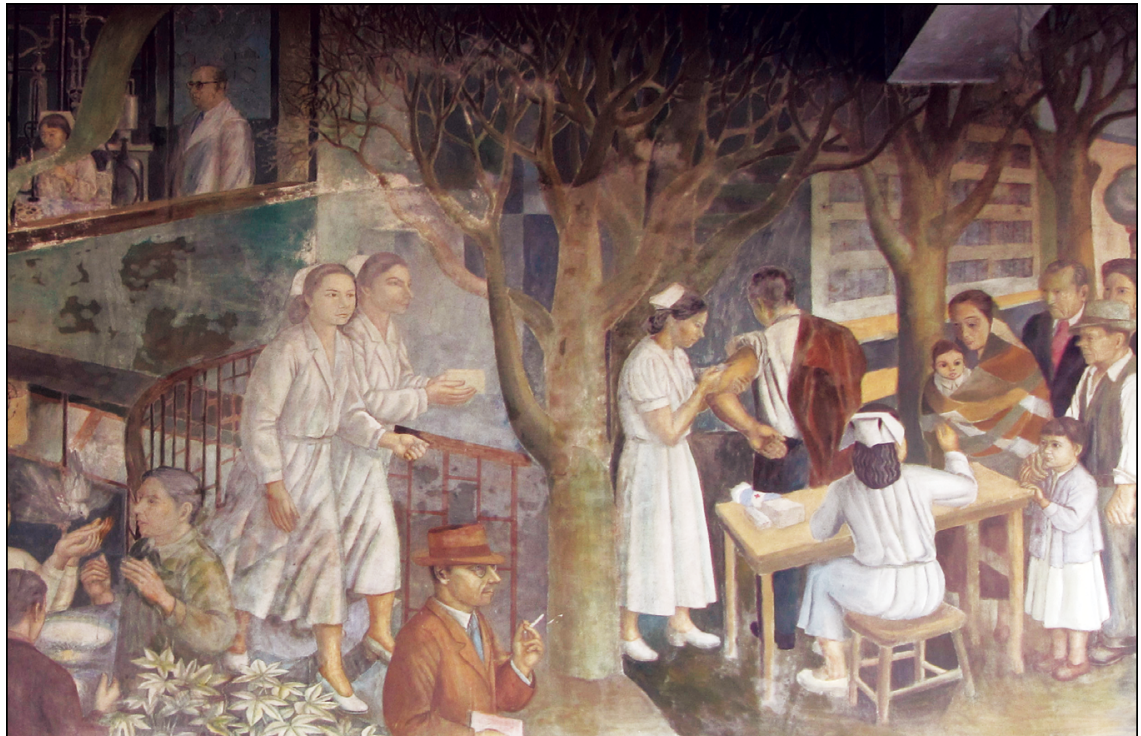

Figura 4b. Medicina moderna detalles.
La instrucción del pintor comenzó en su adolescencia en la Academia de Bellas Artes de Concepción dirigida por el pintor Adolfo Berchenco y fue unos de los ayudantes más jóvenes (18 años) de Gregorio de La Fuente en la ejecución del mural "La historia de Concepción" (1943-45) en la estación de ferrocarriles de esa ciudad ${ }^{12,18,21,22}$. Posteriormente estudio en Santiago en las Escuelas de Bellas Artes y de Artes Aplicadas, Universidad de Chile, donde fue estudiante del muralista Laureano Vergara y del pintor Marco Bonta ${ }^{12,19,21-23}$. Subsecuentemente obtuvo una beca para viajar a Florencia, Italia, (1955-57) con el objeto de estudiar la pintura al fresco, además de viajar por toda Europa, siendo influenciado por la pintura mural del Giotto y de Piero de la Francesca ${ }^{12,18,19,21}$. También estudio en la Academia de Arte de Dusseldorf, Alemania y tuvo estudios en el Museo de Historia del Arte de Viena, y a su regreso a Chile fue nombrado profesor de Pintura Mural del Instituto de Arte, Universidad de Concepción ${ }^{12,18,19,21}$. En sus años en Costa Rica, Escámez trabajo extensivamente pintando murales, haciendo grabados y diseñando numerosas escenografías para obras teatrales (Cervantes, Shakespeare, Boccacio) y para el Ballet Nacional costarricense y además tuvo una intensa y productiva actividad docente, razones por la que fuera declarado Doctor Honoris Causa de la Universidad Nacional de ese país el $2015^{12,19,21-23}$.
Cualquier reseña de la vida del pintor quedaría incompleta sin describir la gran afabilidad que al parecer este tenía, como trasuntan las opiniones de la gente que lo conocieron ${ }^{12,19,21,24}$. Él pudo equilibrar por muchos años una amistad estrecha con P. Neruda y P. de Rokha, a pesar de ser estos dos poetas rivales acérrimos que demandaban gran lealtad de sus amigos, y así fue ilustrador del Arte de Pájaros de Neruda (1973) y de su Fin de $M u n d o^{21,25}$. A pesar de permanecer soltero llevaba una vida sentimental vivaz y uno de sus más notables romances fue aquel de juventud con Violeta Parra, la cual le dedicara unas agudas décimas de despecho y según dicen, lo amenazara públicamente con su guitarra al percatarse de su infidelidad ${ }^{21}$. La bonhomía de Escámez también se manifestó en su facilidad para adaptarse a la vida en Costa Rica, el respeto a su persona y a su obra que despertó en ese país y por las relaciones de amistad generosa que mantuvo con la población rural que lo rodeaba en su casa - taller en Heredia, un suburbio de San José, asiento de la Universidad Nacional ${ }^{21,24}$.

\section{Historia de la Medicina y de la Farmacia en Chile y su contexto}

Además de la solicitud de la propietaria de la farmacia, doña Maria Maluje, es difícil discernir de donde obtuvo la idea el pintor para ejecutar este 
mural de contenido médico y farmacéutico. Existe información que indica que el alterno con Diego Rivera en Concepción, a mediados de los 1950 y tal vez debe haber oído de la boca de este, que el recién había pintado en el Hospital La Raza en Ciudad de México su último gran mural "Historia de la medicina en México El pueblo en demanda de salud"11,19,21,26, que Escámez debe haber probablemente visto al visitar ese país, después de haber pintado el suyo ${ }^{11,26}$. Sin embargo, si la idea para el mural puede haber venido parcialmente de Rivera el mural de Escámez escapa a cualquiera similitud estética con el de este artista, y si esta lejanamente existiera, no va más allá de la representación de la medicina aborigen y de la moderna de ambos países, en los dos murales ${ }^{11,26}$.

La tradición de murales con contenidos religiosos y médicos, comienza en el Renacimiento italiano con el realista "Cuidado de los enfermos" de Doménico de Bartolo, pintado en el hospital Santa María de la Escala, en Siena, entre 1441-44 y la "Madona de la Misericordia", pintada en el hospital de la Misericordia en Borgo Santosepolcro, por Piero de la Francesca en 1444-61 y ese tipo de actividad artística continua interrumpidamente hasta hoy en día como lo demuestran el mural "La diosa de la Salud" (1985) de Pedro Olmos en el hospital FUSAT de Rancagua y el mural "Medicina de Indoamérica al tercer milenio" (2004) de Daniel Palominos en la Universidad Católica del Norte $^{27,28,29,30}$. Un análisis del mural de Escámez pareciera encontrar la inspiración parcial para el en un mural alejado de la medicina, pero que al igual que este mural, introduce el paisaje y el pasaje de la historia y la vida de los individuos en su narrativa ${ }^{31-34}$. Este mural sería el de Ambrosio Lorenzetti "Efectos del buen gobierno" pintado entre 1333-40 en la Sala de la Paz, del Palacio Público de Siena, y que es el primer mural en el mundo que introduce en su contenido a la campiña y su perspectiva, como tan acertadamente y originalmente las usa Escámez en la primera sección de este mural ${ }^{18,19,30-34}$. La influencia de la Escuela de Siena de finales de la Edad Media, más que el arte renacentista de Florencia, se aprecia también, porque el pintor usa murallas y edificios para separar e ilustrar diversos aspectos de la historia de la medicina en Chile, y fueron los pintores sieneses del 'trecento' italiano, entre ellos el Duccio di Buoninsegna, los primeros en representar este tipo de arquitectura en sus pinturas ${ }^{31-34}$.
Sin lugar a dudas que el fresco discutido se beneficia de la gran pericia manual y plástica del pintor, pero a uno le queda la sensación que este mural habría sido imposible sin el tremendo acervo cultural de este y de la experiencia adquirida en sus viajes a través del mundo y de la asimilación y la síntesis de esta experiencia para entender la realidad chilena ${ }^{12,18,19}$. A nuestro modo de ver, la genialidad y la originalidad de Escámez está en aquella síntesis artística que, usando dispositivos tradicionales de la pintura elaborados en la Edad Media y el Renacimiento, le permite retratar en su obra a la etnia mapuche y a sus rituales médicos, a la hibrida medicina colonial y a la medicina moderna de una manera novedosa. Esta misma capacidad de síntesis original le permite pintar a intelectuales chilenos como A. Liptchütz y D. Belmar con R. J. Oppenheimer, un intelectual estadounidense perseguido políticamente en la época que se pintó el cuadro ${ }^{20}$. Las raíces de este mural, y de la obra del pintor en general, nos hace recordar la suma poética de Gabriela Mistral y de su amigo P. Neru$\mathrm{da}$, que a pesar de su resonancia internacionales jamás dejan de ser chilenas y que como lo afirmara Neruda "La pintura de Julio Escámez, su carrera estética, son un lujo para nuestro país ${ }^{18 " . ~}$

Agradecimientos: La lectura de la tesis de la Srta. Paula Domínguez Correa, "De los artistas al pueblo: esbozos para una historia del muralismo social en Chile", Universidad de Chile, 2006 e intercambios generosos con ella como así mismo sus fotografías del mural, fueron esenciales para la composición y la publicación de este artículo y se agradecen profundamente. Agradezco al profesor Nain Nomez, Universidad de Santiago, clarificaciones acerca de la obra de Pablo Neruda y de Pablo de Rokha y de sus relaciones con Julio Escámez. El artículo recuerda al químico farmacéutico Carlos Contreras Maluje, hijo de los antiguos propietarios de la farmacia donde se encuentra el mural objeto del artículo, y amigo de mi esposa Lieselotte Aron H., desaparecido en noviembre de 1976 hasta hoy en día.

\section{Referencias}

1. Degenerate Art: The Attack on Modern Art in Nazi Germany 1937. Peters O. Ed. Prestel. New York. 2014.

2. Barron S. Degenerate Art: The Fate of the Avant-Garde 
in Nazi Germany. Harry N. Abrams. New York. 1991.

3. Before the Fall: German and Austrian Art in the 1930s. Peters P. Ed. Prestel. New York. 2018.

4. Brettel R. Modern Art 1851-1929: Capitalism and Representation. Oxford U. Press. Oxford. R. U. 1999.

5. Read H. A Concise History of Modern Painting. Thames and Hudson, New York, 1986.

6. Wolf N. Expressionism. Taschen. Los Angeles. EE.UU. 2004.

7. Lorenz U. Brücke. Taschen. Los Angeles. EE.UU. 2008.

8. Bitsori M, Galanakis E. Doctors versus artists: Gustav Klimt's Medicine. BMJ 2002; 325 (7378): 1506-8. PubMed PMID: 12493684; PubMed Central PMCID:PMC139056.

9. Finn BC, Bruetman JE, Young P. [The portrait of medicine and Gustav Klimt (1862-1918)]. Rev Med Chile 2013; 141 (12): 1584-8. doi: 10.4067/S003498872013001200013. Spanish. PubMed PMID: 24728437.

10. Pliego Quijano S. El hombre en la encrucijada: el mural de Diego Rivera en el Centro Rockefeller, México, Museo Diego Rivera-Anahuacalli/Trilce Ediciones. México. 2013.

11. Cabello CF. [Diego Rivera: a great master and a didactic and discerning medical historian]. Rev Med Chile 2014 Nov;142(11):1458-66. doi: 10.4067/ S0034-98872014001100014. Spanish. PubMed PMID: 25694292

12. Domínguez Correa P. De los artistas al pueblo. Esbozos para una historia del muralismo social en Chile. Tesis. Facultad de Artes. Universidad de Chile. 2006.

13. Vidal V. Patrimonio Nacional. El mural de Julio Escámez. Anaquel Austral. 2015. http://virginia-vidal.com/ publicados/cronicas/article_615.shtml.

14. Vidal V. Chávez, Escámez, Venturelli: Memoria Grabada. Anaquel Austral. 2013. http://virginia-vidal.com/ catastro/ensayos/article_545.shtml.

15. Venturelli J. Recuperar el arte en Chile: tarea de todos. El caso de Julio Escámez. 2014. http://derechoshumanosyjusticiaparatodos.blogspot.com/2014/03/recuperar-el-arte-en-chile-tarea-de.html.

16. Mural Historia de la Medicina y Farmacología en Chile. http://www.xn--patrimonioparaniasynios-7hcf.cl/mapa/ mural-historia-la-medicina-y-farmacologia-en-chile.

17. Contreras E. Julio Escámez Carrasco. 2015. http:// blogs.cooperativa.cl/opinion/cultura/20151225192703/ julio-escamez-carrasco/.

18. Muralma.Julio Escamez. https://vimeo.com/271488466.

19. Julio Escámez Carrasco. Biografía. Exposiciones. Museo Nacional Bellas Artes. Santiago, Chile. 2019. https:// docplayer.es/52336678-Julio-escamez-biografia-exposiciones-julio-escamez.html.
20. Cabello CF. [The Great European Georg Friedrich Nicolai: physician and pacifist. Berlin, Germany, 1874Santiago, Chile, 1964]. Rev Med Chile 2013; 141 (4): 535-9. doi: 10.4067/S0034-98872013000400017. Spanish. PubMed PMID:23900377.

21. Varas JM. Los sueños del pintor. Penguin Random House Grupo Editorial Chile. 2012.

22. Zamorano Pérez PE, Cortez López C. Muralismo en Chile: texto y contexto de su discurso estético. Universum [online]. 2007; 22:254-274. <https:// scielo.conicyt.cl/scielo.php?script $=$ sci_arttext\&pi$\mathrm{d}=\mathrm{S} 0718-23762007000200017 \& \operatorname{lng}=\mathrm{es} \& \mathrm{nrm}=\mathrm{iso}>$. ISSN 0718-2376. http://dx.doi.org/10.4067/S071823762007000200017.

23. Bragassi J. El muralismo en Chile. Una experiencia histórica para el Chile del Bicentenario. Memoria Chilena. 2010. http://www.memoriachilena.gob.cl/602/ articles-123178_recurso_2.pdf.

24. Murcia González V. Julio Escámez Carrasco, un legado iberoamericano de Chile a Costa Rica. 2016. http:// www.ibe.tv/es/canal/elportalvoz/541/Julio-Esc\%C3\%A$1 \mathrm{mez}$-Carrasco-un-legado-iberoamericano-de-Chile-a-Costa-Rica.htm

25. Escámez J. Visiones de la infancia y adolescencia del poeta Neruda 1906-1921. Ediciones U. de Concepción. 2004.

26. Rodríguez-Gómez G, Cabello FC. Diego Rivera, The History of Medicine in Mexico: People's Demand for Better Health, mural in 1953 still current. Rev Chile Ped. 90 (3): 351-5. DOI: 10.32641/rchped.v90i3.1085. 2019.

27. Cork R. The Healing Presence of Art. A History of Western Art in Hospitals. Yale U. Press. New Haven. EE. UU. 2012.

28. Schneider Adams L. Italian Renaissance Art. Westview Press. Boulder, Co. EE.UU. 2000.

29. Zamorano P. Pedro Olmos: lo nuestro bajo forma de poesía visual. Revista Universum. Universidad de Talca. 2019. http://universum.utalca.cl/contenido/index-91/ Pedro_Zamorano.pdf.

30. Daniel Palominos, la identidad local plasmada en murales y esculturas. Revista Mas Región. 2019. http://www. revistamasregion.cl/2015/09/daniel-palominos-la-identidad-local-plasmada-en-murales-y-esculturas/

31. Hyman T. Sienese Painting: The Art of a City-Republic. Thames and Hudson, New York. 2003.

32. Boucheron P, The Power of Images: Siena 1338. Polity Press. Cambridge, U.K. 2018.

33. Wolf N. Landscape painting. Taschen. Los Angeles. EE.UU. 2008

34. Andrews M, Landscape and Western Art. Oxford U. Press. Oxford. R.U. 2000. 